\title{
Referendum gegen die Managed-Care-Vorlage
}

\section{Daniel Bracher}

Für den Verein für freie Arztwahl

Korrespondenz:

Dr. med. Daniel Bracher Präsident Verein für

freie Arztwahl

Walchstrasse 19

CH-3073 Gümligen

Tel. 0794860713

dbracher@datacomm.ch
Liebe Kolleginnen und Kollegen

Vorab danken wir allen, die sich an der Urabstimmung beteiligt haben; denn 15000 abgegebene Stimmen sind eine repräsentative Zahl. Mit der Urabstimmung sind allerdings weder die 50000 Unterschriften gesammelt noch ist die Abstimmung gewonnen, und unsere Vereinigung bittet Sie nun a) beim Sammeln der Unterschriften mitzuhelfen und b) die Reihen in der FMH wieder zu schliessen.

\section{Sammeln der Unterschriften}

Bitte beachten Sie als Erstes und Wichtigstes: die eine bis fünf Unterschriften pro Bogen müssen alle von Stimmberechtigten stammen, die in derselben Gemeinde in eidgenössischen Angelegenheiten stimmberechtigt sind. Bitte tragen Sie also auf jeden Bogen gross und deutlich den Namen und die Postleitzahl der jeweiligen Gemeinde ein. Die Unterschriftenbogen laden Sie von unserer Homepage www.verein-freie-arztwahl.ch herunter. Die Vorderseite der Bogen müssen Sie belassen, hingegen können Sie unseren Vorschlag für die Gestaltung der Rückseite (und damit der Wahl der Argumente) abändern.
Bitte senden Sie die Bögen so rasch wie möglich, spätestens bis 10. November an unser Sekretariat: Vereinigung für freie Arztwahl, Postfach 30, 3122 Kehrsatz. Der Verein für freie Arztwahl bemüht sich, effizient zu arbeiten (freiwilliger empfohlener Jahresbeitrag unserer Mitglieder 30 Franken). Wir sind Ihnen daher dankbar, wenn Sie in einen Umschlag mit 6 Bögen auch 5 Briefmarken zu 85 Rappen legen.

\section{Die Reihen in der FMH schliessen}

Wir planen, den Referendumskampf auf die obligatorische Budgetmitveranwortung (oBmv) und auf den differenzierten Selbstbehalt (diff. SB) auszurichten. Der diff. SB ist kein Anreiz, sondern eine Busse bis maximal 500 Franken, und zwar ausschliesslich für Kranke, welche sich nicht auf oBmv-Experimente einlassen mögen, sondern an der freien Arztwahl festhalten wollen. Die oBmv soll sich im Wettbewerb durchsetzen und nicht durch Wettbewerbsverzerrung, zumal sie die Gefahr der unkoordinierten, verdeckten Rationierung beinhaltet.

Hingegen liegt es uns fern, integrierte Behandlung in Frage zu stellen, aber dazu braucht es schon heute gar keine oBmv.

\section{Referendum contro il progetto di legge concernente il Managed Care}

\section{Daniel Bracher}

Per il Verein für freie Arztwahl

Corrispondenza: Dr. med. Daniel Bracher Presidente Verein fü freie Arztwahl Walchstrasse 19 CH-3073 Gümligen

dbracher@datacomm.ch
Care colleghe e colleghi,

innanzitutto ringraziamo tutti coloro che hanno partecipato alla votazione generale: 15000 voti consegnati sono infatti un numero rappresentativo. Tuttavia con la votazione generale non sono ancora raccolte le 50000 firme né la votazione è vinta e la nostra associazione ora vi chiede a) di collaborare alla raccolta delle firme e b) di ricompattare i ranghi nella FMH.

\section{Raccolta delle firme}

Per favore ponete attenzione a questa prima - e importantissima - questione: le firme, da due a cinque per ogni modulo, devono provenire tutte da persone solo aventi diritto di voto al livello federale nello stesso comune. Perciò iscrivete chiaramente e in grande in ogni modulo il nome e il codice postale del relativo comune.

Scaricate il moduli per le firme dalla nostra homepage www.verein-freie-arztwahl.ch. Dovreste lasciare così come è la pagina anteriore del modulo; per contro potete modificare la nostra proposta per la creazione del retro-pagina (cioè della scelta degli argomenti). Per favore spedite il modulo al più presto possibile al nostro segretariato: Vereinigung für freie Arztwahl, Postfach 30, 3122 Kehrsatz.

L'Associazione per la libera scelta del medico (Verein für freie Arztwahl - Association pour le libre choix du médecin) si sforza di lavorare in modo efficiente (contributo volontario annuo raccomandato per i nostri soci 30 franci). Vi siamo perciò grati se in ogni busta con 6 moduli accludete anche 5 francobolli da 0.85 franci.

\section{Ricompattare i ranghi nella FMH}

Intendiamo focalizzare la battaglia del referendum sulla corresponsabilità budgetaria obbligatoria («obligatorische Budgetmitveranwortung», oBmv) e sulla aliquota percentuale differenziata (quota di partecipazione ai costi a carico del paziente, «differenzierter Selbstbehalt», diff.SB). L'aliquota percentuale differenziata (diff.SB) non è un incentivo bensì una multa fino a un massimo di 500 franci, e precisamente solo per quegli ammalati che vogliono restare fedeli alla libera scelta del medico e non vogliono avere a che fare con sperimentazioni di corresponsabilità di budget. La corresponsabilità budgetaria obbligatoria (oBmv) deve imporsi attraverso la concorrenza e non attraverso una distorsione della stessa, tanto più che contiene il pericolo di un razionamento delle cure scoordinato e mascherato.

Al contrario, siamo ben lontani dal mettere in discussione l'assistenza sanitaria integrata, ma per questo fine non è necessaria già oggi nessuna corresponsabilità budgetaria obbligatoria. 